\title{
La $44^{\mathrm{e}}$ élection présidentielle américaine Le regard des Américains dans un contexte mondial difficile
}

\section{Cynthia Ghorra-Gobin}

\section{(2) OpenEdition}

1 Journals

\section{Édition électronique}

URL : http://journals.openedition.org/echogeo/9113

DOI : 10.4000/echogeo.9113

ISSN : 1963-1197

Éditeur

Pôle de recherche pour l'organisation et la diffusion de l'information géographique (CNRS UMR 8586)

Référence électronique

Cynthia Ghorra-Gobin, «La 44e élection présidentielle américaine

Le regard des Américains dans un contexte mondial difficile », EchoGéo [En ligne], Sur le Vif, mis en ligne le 19 novembre 2008, consulté le 01 mai 2019. URL : http://journals.openedition.org/ echogeo/9113; DOI : 10.4000/echogeo.9113

Ce document a été généré automatiquement le 1 mai 2019.

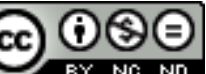

EchoGéo est mis à disposition selon les termes de la licence Creative Commons Attribution - Pas d'Utilisation Commerciale - Pas de Modification 4.0 International 


\title{
La $44^{e}$ élection présidentielle américaine Le regard des Américains dans un contexte mondial difficile
}

\author{
Cynthia Ghorra-Gobin
}

\section{NOTE DE L'AUTEUR}

Ce texte est le deuxième article d'une série de trois articles sur les Etats-Unis rédigé au fur et à mesure des événements à l'occasion de la $44^{\mathrm{e}}$ élection présidentielle. Le premier qui avait pour sous-titre Un événement révélateur des mutations de la société américaine soulignait l'originalité du sénateur candidat à l'élection présidentielle de 2008 en raison de son parcours personnel. Le deuxième article rend compte du regard des Américains qui, à l'occasion de cette élection présidentielle, privilégient les valeurs symboliques véhiculées par le futur président Obama. Le troisième article (à la suite de la prise des fonctions du président le 20 janvier 2009) donnera une idée plus précise de l'orientation que souhaite donner le président à son pays ainsi que de sa vision du rôle des Etats-Unis dans un contexte mondial caractérisé par une grande incertitude.

1 La $44^{e}$ élection présidentielle américaine qui a commencé début janvier dans l'Etat de l'Iowa vient de se traduire par la victoire de Barack H. Obama, un candidat démocrate au parcours atypique. Ce dernier a remporté 364 Grands Electeurs contre 174 pour son adversaire, le candidat républicain John McCain, soit une avance de 190 points $^{1}$. Aux Etats-Unis, le vote des Grands Electeurs (dont le nombre au sein de chacun des 50 Etats varie en fonction du poids démographique) est en effet décisif pour l'élection du président $^{2}$. Le peuple américain s'est prononcé à $52 \%$ en faveur d'Obama (contre $46 \%$ pour McCain) dont l'expérience politique, après tout, est bien limitée ${ }^{3}$. Toutefois pour les Américains, Obama présente, a priori, les qualités requises pour faire face à un contexte national et mondial jugé extrêmement difficile et incertain. Les Etats-Unis sont engagés à 
l'échelle mondiale dans deux guerres et ils font face à une crise financière sans précédant, accompagnée d'un début de récession économique, mettant en évidence une fois de plus la vulnérabilité des populations pauvres, principalement concentrées dans les villes. Mais, si comme le répètent de nombreux Américains «nous avons fait le pari de choisir Obama » - ce qui leur permet d'éviter d'avoir recours à des arguments a priori objectifs et fondés d'un point de vue rationnel - il nous revient d'expliquer la nature de ce pari. Tel est l'objectif de cet article ${ }^{4}$.

\section{Quelques points de repère du vote du 4 novembre 2008}

2 Le mardi 4 novembre 2008, les Américains furent nombreux à se mobiliser : $65 \%$ des électeurs (133,3 millions de personnes) ont voté, ce qui représente un pourcentage relativement important comparé à celui de $50 \%$ généralement évoqué par les politologues pour les dernières décennies. Les médias américains ont souligné que dans certains bureaux de vote, notamment dans les grandes villes, les files d'attente furent longues et ils précisent combien certains électeurs ont dû faire preuve d'une grande patience pour glisser leur bulletin de vote dans l'urne. Le jeune sénateur Obama, un démocrate de 47 ans, ayant moins d'expérience politique que le sénateur McCain, l'a emporté parce que ce dernier (bien que ne s'inscrivant pas dans la lignée directe du président Bush) représentait un parti républicain relativement épuisé (après les huit années de l'administration Bush). Les Américains partagent en effet le sentiment de ne pas se « retrouver sur la bonne voie » et de devoir par ailleurs faire face à des problèmes de grande ampleur chez eux comme à l'étranger 5 .

3 L'élection d'Obama en tant que président des Etats-Unis a bouleversé la cartographie électorale de la décennie 2000, différenciant les Etats colorés en bleu (Etat où la majorité vote pour les démocrates) et les Etats colorés en rouge (Etats où la majorité vote pour les Républicains). La couleur bleu se retrouvant principalement sur les deux côtes de l'Atlantique et du Pacifique et autour des Grands Lacs alors que la couleur rouge rassemble les Etats des grandes plaines centrales et quelques Etats du Sud. Obama a réussi dans les Etats de l'Iowa ${ }^{6}$, de l'Ohio et de l'Indiana ainsi que de la Pennsylvanie, de la Virginie et de la Floride. Ces Etats bien ancrés à droite ont basculé dans le camp démocrate. Dans l'ouest, le Colorado et le Nouveau-Mexique, qui enregistrent le développement d'une industrie high-tech, ont voté pour Obama, ainsi que le Nevada. La ville de Las Vegas concentre en effet des travailleurs latinos qui ont voté pour Obama, ce qui a eu pour conséquence de faire basculer l'Etat dans le champ démocrate ${ }^{7}$. Pour de nombreux observateurs, les habitants de l'Arizona ont voté pour McCain parce qu'il représente l'Etat au Sénat, mais ils reconnaissent que les électeurs risquent de se prononcer pour un candidat démocrate à l'avenir.

Obama a réussi à franchir les frontières partisanes grâce au vote des jeunes, des nouveaux électeurs, et grâce à une forte participation des minorités ethniques. Au niveau national, il a remporté $43 \%$ des votes émanant de Blancs (leur pourcentage est très élevé dans les milieux bénéficiant d'un niveau d'éducation élevé), $66 \%$ des voix des Latinos, $97 \%$ des voix des Africain-Américains, et $67 \%$ des voix des Asiatiques ${ }^{8}$. 


\section{Le nouveau président : un symbole de l'histoire américaine}

Barack Obama, un Américain né en 1961 au sein du couple constitué par une blanche américaine et un jeune étudiant venu du Kenya (pour étudier dans une université américaine) est a priori un métis9 qui a l'expérience d'un vécu à l'étranger avant de se lancer dans des études supérieures le menant ainsi d'Occidental College (située dans l'aire métropolitaine de Los Angeles) à l'Université de Columbia (New York) et ensuite à Harvard ${ }^{10}$. Il entreprend toutefois de construire son parcours politique et professionnel en privilégiant un ancrage dans la communauté africaine-américaine à Chicago : d'abord en tant que community organizer (deux années) et, plus tard, en tant qu'élu au sénat de l'Etat de l'Illinois, avant de devenir sénateur de l'Etat de l'Illinois à la Chambre des Représentants. Cet ancrage dans la communauté africaine-américaine a été largement facilité par son mariage avec Michelle, une avocate brillante ayant grandi dans le ghetto noir de South Park dans la ville de Chicago. Comme l'illustre avec clarté son discours de Philadelphie (18 mars 2008), il connaît bien l'histoire raciale du pays, il fait certes le choix de s'y inscrire mais en la transcendant ${ }^{11}$.

6 En évitant avec habileté de mener une campagne faisant appel au sentiment communautaire (celui des Africains-Américains) et en se proclamant le président des Etats-Unis (et non pas uniquement des Etats bleus), Obama a réussi à symboliser la fin de l'histoire des revendications de la minorité noire qui, après avoir été libérée de l'esclavage, a dû se battre pour obtenir des droits équivalents aux Blancs. Tout au long de sa campagne, et à l'occasion de tous les meetings, il représentait l'image vivante du rêve américain tel qu'il fut incarné par Martin Luther King, il y a plus de quarante cinq ans. Obama représente et condense un siècle d'histoire américaine. Il est la figure rédemptrice d'une Amérique marquée par l'esclavage et la ségrégation et, de ce fait, il est plus qu'un simple candidat démocrate ayant gagné les élections présidentielles, suite à une campagne de qualité ${ }^{12}$.

Obama a en effet mené une campagne-modèle de type on-line et off-line tout en bénéficiant d'un important budget. Il a refusé le financement public et, de ce fait, a réussi à collecter des fonds privés s'élevant à 639 millions de dollars dont 190 ont été dépensés en publicité dans les médias et 66 demeurent encore non utilisés. Sur la liste des principaux contributeurs privés, les employés de Google ont placé leur entreprise au premier rang avec 562241 millions de dollars, suivi par les employés et les professeurs de l'Université de Stanford (Ca) et ceux de l'Université de Californie à Los Angeles (UCLA). Le personnel des universités publiques de Californie a ainsi apporté un montant de l'ordre de 1,3 millions de dollars, un montant uniquement dépassé par l'ensemble des firmes américaines de haute technologie, soit 1,9 millions de dollars ${ }^{13}$.

\section{Le nouveau président : le symbole de l'Amérique des villes}

8 Le rêve américain proposé par les Pères fondateurs reposait sur l'élaboration d'un projet démocratique ancré dans les valeurs du monde rural. Pour Jefferson, tout Américain avait l'opportunité d'accéder à la propriété foncière et de participer à la vie politique. Les 
Etats-Unis ont accepté au début du XIX ${ }^{e}$ siècle d'entrer dans l'ère industrielle (après les Britanniques) et de faire face à l'urbanisation (le corollaire de l'industrialisation) tout en choisissant à ce moment de valoriser l'univers suburbain organisé autour du principe de la maison individuelle entourée d'un jardin pour tous ${ }^{14}$. La banlieue symbolisait ainsi le compromis entre les valeurs de la société rurale et l'impératif économique et la ville fut, tout au long du $\mathrm{XX}^{\mathrm{e}}$ siècle, principalement réduite aux fonctions économiques et à l'acculturation des immigrés. Cette faible valorisation de la ville dans le champ politique se retrouve au niveau du profil des candidats aux élections présidentielles. Ils n'ont jamais eu de responsabilités politiques en tant que maire d'une ville. Ce sont généralement des représentants d'un Etat au Sénat américain ou des gouverneurs. Au moment où le candidat McCain a fait le choix du gouverneur d'Alaska comme co-listière, de nombreux articles et commentaires ont d'ailleurs surgi dans la presse américaine pour faire l'apologie des valeurs de la petite ville. Sarah Palin avait en effet été maire de Wassila (Alaska) avant d'être élue gouverneure.

Obama qui a eu le privilège de vivre dans un grand nombre de villes américaines (et étrangères) ne correspond pas vraiment à l'image traditionnelle d'un candidat issu d'une petite ville ou d'un ranch. Il incarne les valeurs de la ville et du ghetto, et il a été largement plébiscité dans les villes et aires métropolitaines.

Dans une ville comme LA ${ }^{15}, 76 \%$ des électeurs blancs ( $43 \%$ à l'échelle nationale) et $77 \%$ des électeurs latinos ( $66 \%$ à l'échelle nationale) ont voté pour lui (les chiffres sont sensiblement les mêmes pour les Noirs et les Asiatiques). San Fernando (1,2 millions d'habitants), un quartier de la ville de Los Angeles qualifié au début de la décennie 2000 de territoire conservateur parce que certains habitants s'étaient organisés dans des mouvements associatifs pour faire sécession avec la ville de Los Angeles, a voté à $72 \%$ pour Obama. Ce chiffre est légèrement inférieur à celui de $78 \%$ pour l'ensemble de la ville (3,9 millions d'habitants). Par ailleurs les politologues notent que les Latinos sont désormais acquis au parti démocrate : $77 \%$ des Latinos de Californie ont voté pour Obama et $57 \%$ des Latinos de Floride.

11 Au sein des cinq comtés qui constituent l'aire métropolitaine de Los Angeles, le comté urbain de Los Angeles a voté à $69,3 \%$ pour Obama (et seulement $28,8 \%$ pour McCain), le comté de Ventura a voté à $97 \%$ pour Obama, celui de San Bernardino à 49,9\% (contre $47,9 \%$ pour McCain) et celui de Riverside à $50,4 \%$ pour Obama (au lieu de $47,9 \%$ pour McCain). Seul le comté d'Orange, souvent qualifié de réactionnaire parce que concentrant de vastes lotissements fermés (gated communities), a voté pour McCain mais le pourcentage n'est que de 50,8\% alors que Reagan avait obtenu $90 \%$ des voix. Force est d'admettre que les habitants des résidences fermés ont voté tout comme la mainstream urban America.

\section{Conclusion : L'Amérique face à un tournant de son histoire}

Le charisme d'Obama, son sens politique ainsi que ses capacités rhétoriques ont conduit les médias américains à proclamer America Makes History (une expression rarement utilisée au cours des dernières décennies) pour ensuite écrire Obama wins. La campagne d'Obama a démontré qu'il était en mesure de convaincre le peuple américain, en ne 
cessant de lui répéter qu'il pouvait dépasser ses divisions pour œuvrer ensemble. Ce fut la force du leitmotiv "yes we can" qui dans certains quartiers fut traduit par "si se puede".

Clore un chapitre de l'histoire raciale américaine et incarner l'Amérique des villes, deux symboles portés par un candidat tout au long de sa campagne présidentielle, exige toutefois quelques éclaircissements quand il s'agit d'expliquer sa victoire. Obama a bénéficié de circonstances exceptionnelles: un pays confronté à deux guerres, une crise financière d'une grande ampleur, un début de récession et la perspective d'une planète en péril (changement climatique, etc.). En guise d'illustration, rappelons que le candidat a su mettre à profit l'erreur de son adversaire qui affirma le 15 septembre 2008 que les "fondamentaux de l'économie américaine étaient tout compte fait solides» alors que quelques mois auparavant il avait avoué au public que l'économie n'était pas vraiment son point fort.

\section{NOTES}

1. . Le chiffre des grands électeurs peut légèrement varier en fonction de la source utilisée.

2. . La spécificité des élections présidentielles américaines a été remarquablement expliquée à l'opinion publique par les médias internationaux au cours des derniers mois.

3. . Obama est le premier démocrate depuis Jimmy Carter (50,1\% des voix en 1976) à gagner plus de $50 \%$ du vote populaire.

4. . Il aurait certes été possible de se lancer dans une analyse spatiale du résultat des votes en étudiant chacun des 50 Etats ou encore de s'interroger sur la pertinence des hypothèses véhiculées par le courant post-colonial mais ces analyses auraient exigé d'une part de disposer d'une distance temporelle avec l'événement et d'autre part de bénéficier d'un nombre de signes supérieurs à ceux imposés pour l'exercice. Aussi cet article se limite-t-il à rendre compte à privilégier tout simplement le regard des Américains, à la suite d'entretiens menés au mois de septembre 2008 dans quelques villes américaines et au téléphone, une fois les résultats connus .

5. . Les Américains utilisent fréquemment la phrase "we feel we are on the wrong track» et ils s'attendent à faire face à des difficultés supérieures à celles connues lors du krack de Wall Street en 1929.

6. . Le choix de l'Iowa en faveur du candidat Obama est remarquable quand on sait que moins de $3 \%$ de sa population est africaine-américaine.

7. . Pour visualiser la carte des élections, consulter parmi les nombreux sites Internet, www.realclearpolitics.com

8. . Cette répartition des votes en différents groupes ethniques et raciaux reprend les catégories du recensement relevant de l'administration fédérale et reposant sur un compromis politique.

9. . Le terme métis est la traduction française de l'adjectif biracial utilisé par les Américains.

10. . Obama est le deuxième président des Etats-Unis qui a commencé ses études supérieures à l'Ouest des Rocheuses, notent les universitaires américains. Nixon fut le premier.

11. . Ce discours, en raison de son importance, a fait l'objet d'un ouvrage en anglais et en français chez Grasset au printemps 2008, De la race en Amérique. 
12. . L'histoire raciale aux Etats-Unis a fait l'objet de nombreux ouvrages mais celui de l'écrivain Russell Banks se présente comme une excellente synthèse en langue française, Amérique, notre histoire, Actes sud, 2006.

13. . Ces chiffres sont donnés par le Federal Election Commission.

14. . Consulter, Villes et société urbaine aux Etats-Unis, Colin, 2003.

15. . Los Angeles, deuxième ville des Etats-Unis est perçue comme une ville d'avant-garde sur le plan politique parce qu'elle fut la première à voter pour un maire noir alors que les AfricainsAméricains ne représentent que $13 \%$ de la population et depuis 2005, elle est dirigée par un maire hispanique. Los Angeles est la ville américaine préférée de l'auteur qui continue d'y mener un travail de terrain. Los Angeles et Paris ont servi d'études de cas dans le cadre d'une thèse d'Etat privilégiant la démarche comparative pour mettre en évidence les invariants de la ville (Université de Paris 1, 1985). Les chiffres sont empruntésà une étude menée par le Leavey Center for the Study of LA de l'Université Loyola Marymount.

INDEX

Mots-clés : élections présidentielles, Etats-Unis, histoire raciale, mondial, représentations, symbole, valeurs urbaines

\section{AUTEUR}

\section{CYNTHIA GHORRA-GOBIN}

Cynthia Ghorra-Gobin est géographe et américaniste. Elle a un doctorat d'Etat en géographie (Université de Paris 1) et un Ph.D. d'urbanisme (UCLA). Elle est affiliée au CNRS où elle est directeur de recherche et est professeur à l'Université de Paris IV et à l'Institut d'Etudes Politiques (Paris). Ses travaux de recherches abordent les questions urbaines, sociales ethniques et culturelles à partir d'un travail de terrain mené aux Etats-Unis. Depuis une dizaine d'années, ils sont centrés sur la thématique de la métropolisation, révélateur de l'articulation entre le global et le local. Parmi ses récentes publications, Les Etats-Unis entre local et mondial (Presses de Sciences Po, 2000), Villes et société américaine (Colin, collection U, 2003) et la direction du Dictionnaire des mondialisations (Colin, 2006). 